\title{
AXIN2 Polymorphisms, the $\beta$-Catenin Destruction Complex Expression Profile and Breast Cancer Susceptibility
}

\author{
Andres Felipe Aristizabal-Pachon ${ }^{1 *}$, Thais Inacio Carvalho ${ }^{2}$, Helio Humberto \\ Carrara $^{3}$, Jurandyr Andrade ${ }^{3}$, Catarina Satie Takahashi ${ }^{1}$
}

\begin{abstract}
Background: The Wnt/ק-catenin signaling pathway is an important regulator of cellular functions such as proliferation, survival and cell adhesion. Wnt/ $\mathbf{\beta}$-catenin signaling is associated with tumor initiation and progression; $\beta$-catenin mutations explain only $30 \%$ of aberrant signaling found in breast cancer, indicating that other components and/or regulation of the Wnt/ $\beta$-catenin pathway may be involved. Objective: We evaluated AXIN2 rs2240308 and rs151279728 polymorphisms, and expression profiles of $\beta$-catenin destruction complex genes in breast cancer patients. Materials and Methods: We collected peripheral blood samples from 102 breast cancer and 102 healthy subjects. The identification of the genetic variation was performed using PCR-RFLPs and DNA sequencing. RT-qPCR was used to determine expression profiles. Results: We found significant association of AXIN2 rs151279728 and rs2240308 polymorphisms with breast cancer risk. Significant increase was observed in AXIN2 level expression in breast cancer patients. Further analyses showed APC, $\beta$-catenin, CK1 $\alpha$, GSK3 $\beta$ and PP2A gene expression to be associated to clinic-pathological characteristics. Conclusions: The present study demonstrated, for the first time, that AXIN2 genetic defects and disturbance of $\beta$-catenin destruction complex expression may be found in breast cancer patients, providing additional support for roles of Wnt/ß-catenin pathway dysfunction in breast cancer tumorigenesis. However, the functional consequences of the genetic alterations remain to be determined.
\end{abstract}

Keywords: AXIN2 - beta-catenin destruction complex - breast cancer - SNP - RFLP-PCR - gene expression - RT-qPCR

Asian Pac J Cancer Prev, 16 (16), 7277-7284

\section{Introduction}

Breast cancer is one of the most common diseases suffered by women around the world, with an estimated 990,000 new cases and 375,000 deaths each year (Brito et al., 2009). In the last report of IARC (International Agency for Research on Cancer), for 2030 there will be 27 million new cases of cancer diagnosed in the world, 75 million people will have the disease and 17 million will die each year. In Brazil, breast cancer is among the most important cause of death since 1979 (Brito et al., 2009). In 2014, new cases were 49,400, with an estimated risk of 50.1 cases for every 100,000 women. Breast cancer is considered the most common cancer on the population, accounting for $28 \%$ of new cancer patients, furthermore, is the seventh leading cause of death in Brazil after vascular disease and diabetes, overcoming the other cancers, and it is responsible of $2.3 \%$ of deaths in the country (INCA, 2014), considered as a public health problem and classified as an epidemic. High mortality is caused by its capacity to generate metastasis, being the main targets lung, liver and bone (Teixeira et al., 2009; Santos et al., 2010). Breast cancer is the main cause of death in women between 35 and 64 years old in Brazil and South America, new research efforts aimed at identifying mechanisms and developing tools for early disease detection; in this way, in recent years, many studies have shown that breast cancer is a heterogeneous disease, including a variety of molecular subgroups based on differential expression patterns (Alanazi et al., 2013). Gene expression and epigenetic changes are part of signaling pathways, like Wnt pathway, have been considered in the etiology of breast cancer (Klarmann et al., 2008).

Wnt pathway deregulation including both, components and regulators, has been linked to many cancers, such as melanoma, adrenocortical, liver, colorectal and breast cancer (Lustig et al., 2002; Castiglia et al., 2008; Gunes et al., 2009; MacDonald et al., 2009; Chapman et al., 2011; Herbst et al., 2014). This conserved pathway regulates migration, apoptosis, morphogenesis, differentiation and cell proliferation processes by $\beta$-catenin transcriptional function (Mosimann et al., 2009; Clevers and Nusse, 2012; Herbst et al., 2014; Rennoll et al., 2014). Wnt binds to extracellular domain of Frizzled and LDL receptor-related

${ }^{1}$ Department of Genetics, ${ }^{3}$ Department of Gynecology and Obstetrics, Ribeirao Preto Medical School, University of Sao Paulo, ${ }^{2}$ Department of Oncology, Hospital Santa Casa of Ribeirao Preto, Ribeirao Preto, Brazil *For correspondence: sagipe07@gmail. com,afaristizabal@usp.br 
proteins (LRP5 or LRP6), binding induced Dishevelled (DVL) activation and blocking $\beta$-catenin degradation. Wnt pathway leads to $\beta$-catenin level rise in the cytoplasm and increased entry into the nucleus. $\beta$-Catenin forms a transcription factor complex with T-cell factor/lymphoid enhancing factor (TCF/LEF1) and binds Wnt responsive DNA elements (WREs) to activate target gene expression (Moon et al., 2004; Mosimann et al., 2009; Clevers and Nusse, 2012). In absence of Wnt ligand, $\beta$-catenin destruction complex is assembled by scaffold protein Axis Inhibitor (AXIN), Adenomatous Polyposis Coli (APC), Glycogen Synthase Kinase (GSK3 $\beta$ ) and protein phosphatase 2A (PP2A). This complex coordinates $\beta$-catenin phosphorylation by Casein Kinase (CK1 $\alpha)$ and Glycogen Synthase Kinase 3 (GSK3 $\beta$ ), leading to ubiquitination by ubiquitin ligase protein $(\beta \operatorname{TrCP})$ and subsequently degraded in proteasome.

One of the Wnt pathway target genes is AXIN2, suggesting that AXIN2 gene expression is part of a negative feedback loop in response to $\beta$-catenin level rise in the cytoplasm (Jho et al., 2002; Lustig et al., 2002; Salahshor and Woodgett, 2005). Genetic alterations in AXIN2 generate destruction complex instability and facilitate release of $\beta$-catenin, thereby blocking the negative feedback loop and Wnt pathway regulation. AXIN2 mutations can lead to different cancer due to increase of Wnt target genes transcription (Lammi et al., 2004; Salahshor and Woodgett, 2005; Mostowska et al., 2006; Gunes et al., 2009; Han et al., 2014; Rennoll et al., 2014).

The aim of the present study was to evaluate the association of AXIN2 gene mutations with breast cancer; furthermore, we determined the expression profile of $\beta$-catenin and degradation complex genes in breast cancer patients and healthy control group.

\section{Materials and Methods}

\section{Subjects}

Peripheral blood of 204 females was collected from Santa Casa and Clinics Hospitals of Ribeirao Preto, Sao Paulo University (Brazil). We analyzed 102 breast cancer patients, before chemotherapy or radiotherapy, and 102 healthy controls, without family history of cancer. Breast cancer females were included without restriction of age and pathological stage. Hormone receptor status, histological type and TNM were performed by the histological and immunohistochemistry evaluation of tumor biopsies. Information about parity, age at menarche, menopausal and smoking status, oral contraceptive use and hormone replacement therapy were obtained by specific questionnaire. The control group was healthy voluntaries matched to breast cancer patients by age, and another factors risk (Table 1). Clinical characteristics of breast cancer patients are presented in Table 2.

\section{Ethics statement}

All procedures of this study were approved by Comitê Nacional de Ética em Pesquisa - CONEP number approbation CAAE: 01779812.9.0000.5440 and the Research Ethics Committee (CEP) of Clinics Hospital of
Ribeirao Preto, University of Sao Paulo. Written informed consent was obtained from all participating patient and healthy control.

\section{DNA extraction}

Genomic DNA was isolated from peripheral blood mononuclear cells (PBMCs) by salt extraction, using

Table 1. General Characteristics of Healthy Controls and Breast Cancer Patients

\begin{tabular}{|c|c|c|c|c|c|}
\hline & \multicolumn{2}{|c|}{$\begin{array}{c}\text { BCP } \\
(n=102)\end{array}$} & \multicolumn{2}{|c|}{$\begin{array}{l}\text { Controls } \\
(\mathrm{n}=102)\end{array}$} & \multirow[t]{2}{*}{$\mathrm{p}$-value } \\
\hline & $\mathrm{n}$ & $\%$ & $\mathrm{n}$ & $\%$ & \\
\hline Age (years) Mean \pm SD & \multicolumn{2}{|c|}{$58.16 \pm 12.83$} & \multicolumn{2}{|c|}{$53.51 \pm 11.52$} & $0.407^{1}$ \\
\hline \multicolumn{6}{|c|}{ Smoking } \\
\hline Yes & 23 & 22.5 & 24 & 23.5 & \multirow[t]{2}{*}{$0.868^{2}$} \\
\hline No & 79 & 77.5 & 78 & 76.5 & \\
\hline \multicolumn{6}{|l|}{ Age at menarche $\dagger$} \\
\hline$\leq 13$ & 71 & 69.6 & 78 & 76.5 & \multirow[t]{2}{*}{$0.269^{2}$} \\
\hline$>13$ & 31 & 30.4 & 24 & 23.5 & \\
\hline \multicolumn{6}{|l|}{ Menopause Status } \\
\hline Yes & 86 & 76.1 & 75 & 82.4 & \multirow[t]{2}{*}{$0.059^{2}$} \\
\hline No & 16 & 23.9 & 27 & 17.6 & \\
\hline \multicolumn{6}{|l|}{ OCU } \\
\hline Yes & 39 & 38.2 & 57 & 54.5 & \multirow[t]{2}{*}{$0.012^{2}$} \\
\hline No & 63 & 61.8 & 45 & 45.5 & \\
\hline \multicolumn{6}{|l|}{ MHTU } \\
\hline Yes & 22 & 21.6 & 17 & 16.7 & \multirow[t]{2}{*}{$0.373^{2}$} \\
\hline No & 80 & 78.4 & 85 & 83.3 & \\
\hline \multicolumn{6}{|l|}{ Parity† } \\
\hline$\leq 30$ & 32 & 31.4 & 46 & 45.1 & \multirow[t]{3}{*}{$0.075^{2}$} \\
\hline$>30$ & 50 & 49 & 35 & 34.3 & \\
\hline Nulliparous & 20 & 19.6 & 21 & 20.6 & \\
\hline
\end{tabular}

$\dagger$, Age in years old;; OCU, Oral Contraceptive Use; MHTU, Menopausal Hormone Therapy Use. p-values were calculated by 1 Student's t-test and 2 Chi-square test; $p<0.05$ was considered significant and are depicted in bold

Table 2. Clinical Characteristics of Breast Cancer Patients

\begin{tabular}{llr}
\hline Variable & & $\begin{array}{r}\text { BCP, No.(\%) } \\
\mathrm{n}=102\end{array}$ \\
\hline Histological type & IDC & $80(78.4)$ \\
Tumor Stage & Others & $22(19.6)$ \\
& I & $33(32.3)$ \\
& II & $40(39.2)$ \\
Tumor Size & III & $18(17.7)$ \\
& IV & $11(10.8)$ \\
Lymph Node Status & T1 & $45(44.1)$ \\
& T2 & $40(39.2)$ \\
Estrogen Receptor Status & T3 & $17(16.7)$ \\
& Positive & $57(36.7)$ \\
Progesterone Receptor Status & Negative & $48(47.1)$ \\
& Positive & $90(88.2)$ \\
& Negative & $9(8.8)$ \\
HER2/neu Status & NI & $3(2.9)$ \\
& Positive & $84(82.4)$ \\
& Negative & $15(14.7)$ \\
& NI & $3(2.9)$ \\
& Positive & $22(21.6)$ \\
& Negative & $69(67.6)$ \\
& NI & $11(10.8)$ \\
\hline
\end{tabular}

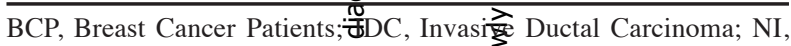
Not Information 
Wizard Genomic DNAPurification Kit (Promega, Madison, WI, USA) and following the manufacturer's instructions. DNA isolated was quantified by spectrophotometer NanoVue (GE, Fairfield, CT, USA), the examination of its purity with $260 / 230$ and $260 / 280$ ratios and integrity by agarose gel electrophoresis.

\section{Genotyping}

The AXIN2 rs2240308 and rs 151279728 polymorphisms were analyzed in genomic DNA from peripheral blood samples of every subject. We determined the AXIN2 c.148C >T (rs2240308) SNP polymorphisms by polymerase chain reaction-restriction fragment length polymorphism (PCR-RFLP). AXIN2 c.2013_2024del (rs151279728) polymorphism was detected by specific PCR amplification and confirmed by direct sequencing. PCR primers used for rs 2240308 polymorphism were forward 5'-CCACGCCGATTGCTGAGAGG-3' and reverse 5'-TTCCGCCTGGTGTTGGAAGAGACAT-3' (Gunes et al., 2009), and rs151279728 polymorphism were forward 5'-TCTCCAGGCGAACGAGCCAG-3' and reverse 5'- ACCTCAGCTAGCCTGCGACA -3' (Castiglia et al., 2008). PCR was performed in a total reaction volume of $20 \mu \mathrm{L}$ containing $100 \mathrm{ng}$ of genomic DNA, 10 pmol of appropriated primer, $0.2 \mathrm{mM}$ of dNTP (GE, Fairfield, CT, USA), $2 \mathrm{mM}$ of $\mathrm{MgCl} 2$ and $1 \mathrm{U}$ of Platinum Taq Polymerase in $1 \mathrm{X}$ buffer (Invitrogen, Carlsbad, CA, USA). PCR conditions for rs 2240308 contained an initial denaturation at $95^{\circ} \mathrm{C}$ for $2 \mathrm{~min}$, followed by 35 cycles of $94^{\circ} \mathrm{C}$ for $1 \mathrm{~min}, 64^{\circ} \mathrm{C}$ for $1 \mathrm{~min}$ and $72^{\circ} \mathrm{C}$ for $1 \mathrm{~min}$, and a final extension at $72^{\circ} \mathrm{C}$ for 5 min. To detect rs2240308 SNP polymorphism, PCR product was digested with $10 \mathrm{U}$ of restriction enzyme Mph1103I (Fermentas, Waltham, MA, USA) in a final volume of $20 \mu \mathrm{L}$; containing $1 \mathrm{X}$ reaction buffer (supplied with the enzyme) for $37^{\circ} \mathrm{C}$ overnight, according to the manufacturer's instructions. Following digestion, DNA fragments were separated on $2.5 \%$ agarose gel. The C allele (wild type) was visualized as two fragments (218 and $24 \mathrm{bp}$ ), whereas T allele yielded just one fragment (242 bp). To identified rs151279728 polymorphism, AXIN2 exon 8 was amplified under following PCR conditions, initial denaturation at $95^{\circ} \mathrm{C}$ for $5 \mathrm{~min}$, followed by 35 cycles at $94^{\circ} \mathrm{C}$ for $45 \mathrm{sec}, 62^{\circ} \mathrm{C}$ for $30 \mathrm{sec}$ and $72^{\circ} \mathrm{C}$ for $45 \mathrm{sec}$, and lastly final extension at $72^{\circ} \mathrm{C}$ for $7 \mathrm{~min}$. PCR product was visualized by $2.5 \%$ agarose gel. The normal allele was visualized as $167 \mathrm{bp}$ band and the deletion c.2013_2024del (rs151279728) was identified for 155 bp band. For quality control, some samples were repeated PCR and sequenced their product.

\section{RNA extraction}

PBMCs were separated with Histopaque 1077 (St. Louis, MO, USA), total RNA was extracted using Trizol (Invitrogen, Carlsbad, CA, USA) according to manufacturer's instructions. The RNA isolated was quantified by spectrophotometer NanoVue (GE, Fairfield, CT, USA), the examination of its purity with 260/230 and 260/280 ratios, and integrity was assessed with RNA 6000 Nano Chip Kit on Bioanalyzer 2100 (Agilent, Santa Clara, CA, USA).

\section{Real time quantitative PCR (RQ-PCR)}

From $1 \mu \mathrm{g}$ of total RNA was synthesized cDNA using DNAse I and SuperSript III Reverse Transcriptase (Invitrogen, Carlsbad, CA, USA). The qPCR was performed using Taqman Universal PCR Master Mix and Taqman inventoried probes on Step One Plus RealTime PCR Systems (Applied Biosystems, Carlsbad, CA, USA) following manufacturer's instructions. The following commercial available TaqMan probes were used, Hs99999901_s1 (18S), Hs01060665_g1 (ACTB), Hs00181051_m1 (APC), Hs00610344_m1 (AXIN2), Hs00793391_m1 (CK1 $\alpha)$, Hs00355049_m1 ( $\beta$-Catenin), Hs02758991_g1 (GAPDH), Hs00275656_m1 (GSK3 $\beta$ ) and Hs00427259_m1 (PP2A). The expression levels were analyzed by threshold cycle $(\mathrm{Ct})$, as recommended by Schmittgen and Livak (Schmittgen and Livak, 2008) with 2- $\Delta \mathrm{Ct}$ method with equipment software DataAssist v3.01 (Applied Biosystems, Carlsbad, CA, USA). GAPDH, ACTB and 18S were used as housekeeping genes. The samples were loaded in triplicate.

\section{Statistical analyses}

Data analysis was carried out with SPSS software version 22 for Windows (Chicago, IL, USA). The observed genotypes were computed and tested for HardyWeinberg equilibrium using Pearson goodness-of-fit chi-square test. Chi-square and Fisher's exact test were used to evaluate the diversity of demographic variables as well as the allele's frequencies and genotypes of the studied polymorphisms between groups. Conditional logistic regression model was used to calculate the odds ratio (OR) and 95\% confidence intervals $(\mathrm{CI})$ for associate the polymorphisms and the risk of breast cancer. The RQPCR results were analyzed by fold-change calculation, it was considered differential expression with fold-change $<-1.5$ or $>1.5$ and adjusted $\mathrm{P}$-value $<0.05$ by BenjaminiHochberg method. Student's t-test and one-way ANOVA analysis were used to determine the significance between groups. P-values $<0.05$ were considered statistically significant and all statistical tests were 2 -sided.

\section{Results}

Association between rs2240308 and rs 151279728 polymorphisms and breast cancer risk

We evaluated rs2240308 SNP polymorphism (c.148 $\mathrm{C}>\mathrm{T}$ ) in 102 breast cancer patients and 102 healthy controls, as shown in table 3; the genotype frequencies $(\mathrm{CC}=19.6 \%, \mathrm{CT}=56.9 \%, \mathrm{TT}=23.5 \%$ and $\mathrm{CC}=43.1 \%$, $\mathrm{CT}=53.8 \%, \mathrm{TT}=2.9 \%$, respectively) was computed and tested for Hardy-Weinberg equilibrium ( $p>0.05$ ), providing no evidence of population stratification. The genotypes distribution of rs2240308 polymorphism was significantly different between breast cancer patients and control group $\left(\mathrm{X}^{2}=25.472 \mathrm{df}=2, \mathrm{p}<0.001\right)$. Homozygous ancestral allele (CC) was used as reference to calculate the risk for developing breast cancer in relation to the other two genotypes. Table 3 shows the genotype distribution of rs2240308 polymorphism (c.148 C>T) with its corresponding odds ratios (OR) and significance. The genotype analysis in breast cancer patients indicated a 


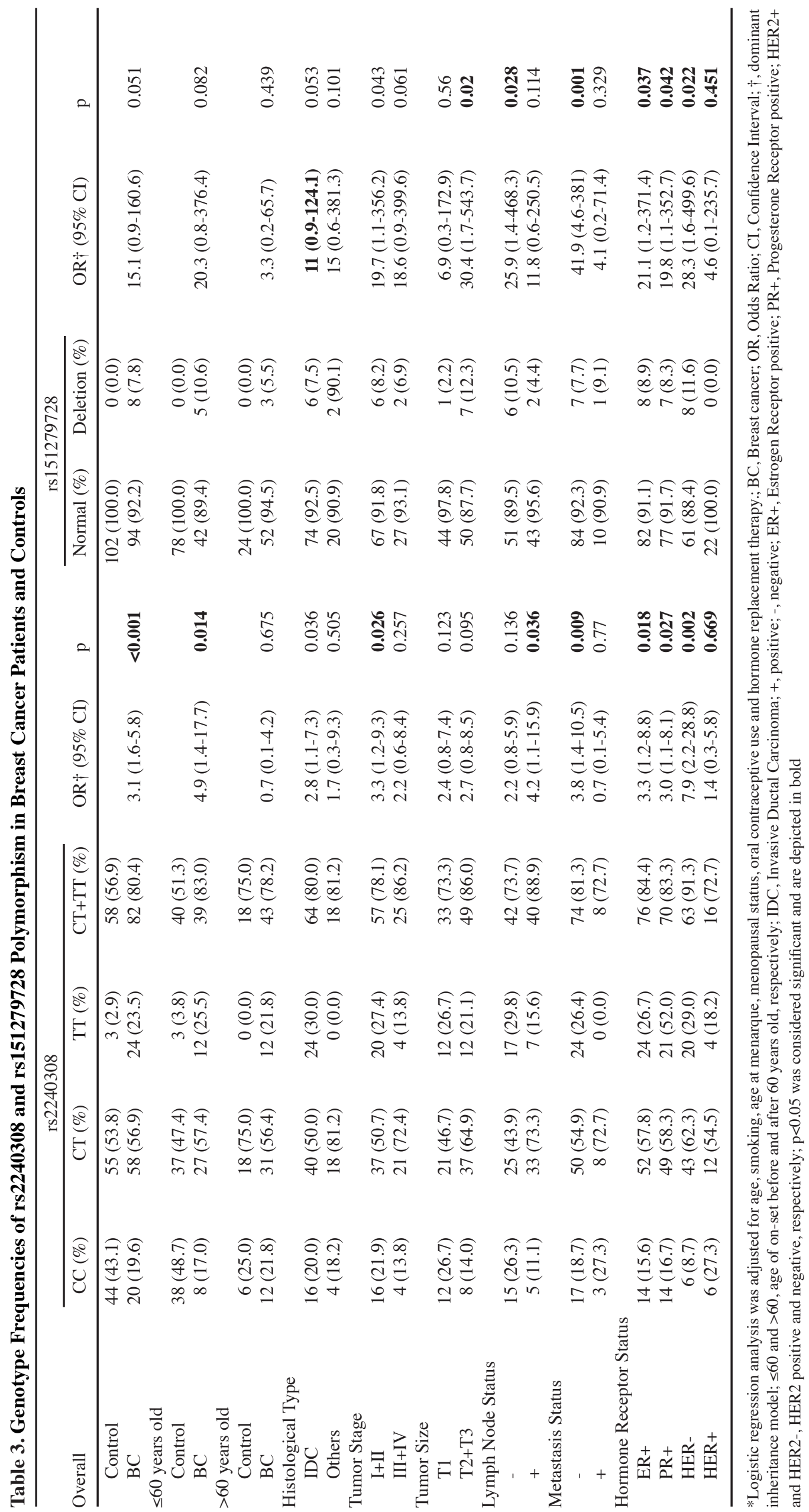

7280 Asian Pacific Journal of Cancer Prevention, Vol 16, 2015 
significant association of rs2240308 polymorphism with breast cancer risk. The presence of $\mathrm{T}$ allele (CT or TT genotype) increasing the risk for developing breast cancer, compared to subjects with wild genotype $\mathrm{CC}(\mathrm{OR}=3.1$; $\mathrm{CI}=1.6-5.8 ; \mathrm{p}<0.001)$.

The AXIN2 exon 8 was amplified to identified rs 151279728 polymorphism; an in-frame 12 bp deletion (c.2013_2024del12), generating a loss of 4 amino acids residues 672-675 (delTTPR). A total of 204 women (102 breast cancer patients and 102 healthy controls) were genotyped for this polymorphism. The deletion was observed in breast cancer patients (Table 3), which is noteworthy, was not found it in the control group. From 102 breast cancer patients, $3(2.9 \%)$ cases were heterozygous and $5(4.9 \%)$ cases had deletion in both alleles. The rs 151279728 polymorphism distribution was significantly different between healthy controls and breast cancer patients $\left(\mathrm{X}^{2}=9.32, \mathrm{df}=2, \mathrm{p}=0.009\right)$. Normal allele (with no deletion) was used as reference to calculate the breast cancer risk in relation to the polymorphism. Table 3 shows the rs 151279728 polymorphism distribution with its respective OR and significance.

To identify the effect of young age at diagnosis on the association of AXIN2 polymorphisms with breast cancer, we divided the cases group by median age of onset (60 years old). We stratified the cases group as patients $\leq$ $60(46.1 \%)$ or $>60(53.9 \%)$ years old. The rs 2240308 polymorphism was associated with increased risk only in the young subgroup ( $\leq 60$ years old), as well as was found in the overall study; older patients ( $>60$ years old) with $\mathrm{T}$ allele did not show significant association with breast cancer risk (Table 3). The rs151279728 polymorphism was not associated with increased risk of breast cancer in relation to age of onset. Among the group of breast cancer patients, we stratified by histological type as invasive ductal carcinoma - IDC (78.4\%), or others carcinomas, including invasive lobular carcinoma - ILC, ductal carcinoma in situ and invasive breast papillary carcinoma (19.6\%); tumor stage as early-stage I - II (71.6\%) or advance-stage III - IV (28.4\%); tumor size as T1 (44.1\%) or T2 + T3 $(55.9 \%)$; lymph node status as positive $(44.1 \%)$ or negative $(55.9 \%)$; and hormone receptor status. Each subgroup was separately compared with the control group to determine the effect of histo-pathological characteristics on the association of AXIN2 variants with the breast cancer. We did not conduct analyses for the ER- and PR- subgroups because of limited sample size. A logistic regression analysis revealed the rs 2240308 polymorphism was significantly associated with risk in IDC patients $(\mathrm{OR}=2.8 ; \mathrm{CI}=1.1-7.3 ; \mathrm{p}=0.036)$, early-stage $(\mathrm{OR}=3.3 ; \mathrm{CI}=1.2-9.3 ; \mathrm{p}=0.236) \mathrm{ER}+(\mathrm{OR}=3.3 ; \mathrm{CI}=1.2-$ 8.8; $\mathrm{p}=0.018), \mathrm{PR}+(\mathrm{OR}=3.0 ; \mathrm{CI}=1.1-8.1 ; \mathrm{p}=0.027)$, HER2- $(\mathrm{OR}=7.9 ; \mathrm{CI}=2.2-28.8 ; \mathrm{p}=0.002)$ and lymph node positive subgroups $(\mathrm{OR}=4.2 ; \mathrm{CI}=1.1-15.9 ; \mathrm{p}=0.036)$, but not in others carcinomas, advance tumor stage and HER2+ subgroups. The tumor size subgroups were not associated the rs 2240308 polymorphism with risk to breast cancer. The rs 151279728 polymorphism was significantly associated with risk in early-stage, tumor larger size, ER+, PR+ and HER2- subgroups. The genotype distribution with odds ratio, confidence interval and significance p-value are shown in Table 3.

Gene expression profile of the $\beta$-catenin destruction complex in breast cancer patients

We evaluated the APC, AXIN2, CK1 $\alpha, \beta$-catenin, GSK3 $\beta$ and PP2A gene expression level in 102 breast cancer patients and 102 normal healthy controls. Figure 1 shows the fold-change (FC) value for every gene in breast cancer patients compared to control group. Significant increase was observed in AXIN2 gene expression (2.33fold, $\mathrm{p}=0.0348$ ) in breast cancer patients.

We explored the association between gene expression profile and histo-pathological characteristics. The others carcinomas subgroup showed decrease expression level compared with IDC subgroup for $\mathrm{CK} 1 \alpha(\mathrm{p}=0.007)$ and PP2A ( $\mathrm{p}=0.002)$ genes (Figure 2.a and 2.b, respectively). Concerning tumor stage, we found differential expression profile between early and advance-stage. Significant decrease was observed for APC $(p=0.003)$ and GSK3 $\beta$

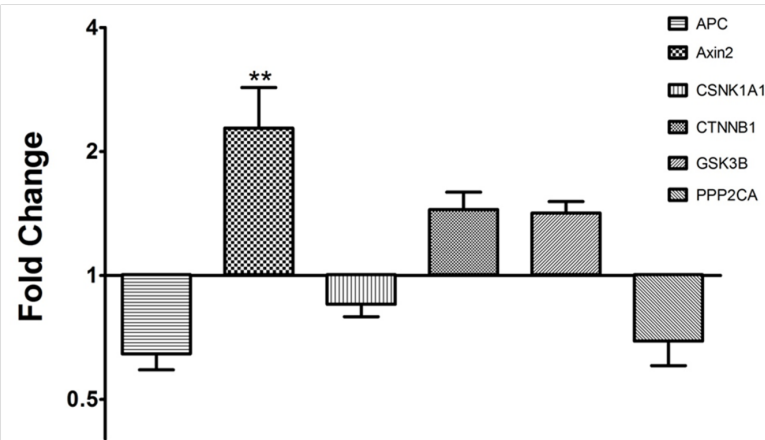

Figure 1. Relative Gene Expression Profile of the $\beta$-catenin Destruction Complex in Breast Cancer Patients. **, Indicate significance difference between breast cancer patients and control group, $\mathrm{p} \leq 0.05$
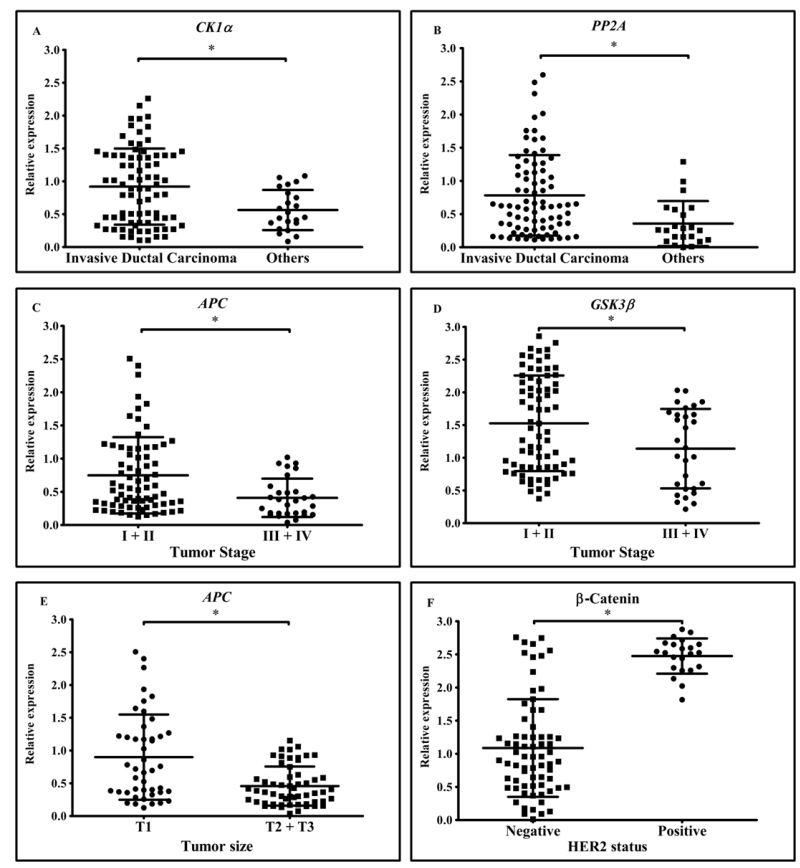

Figure 2. Relative Gene Expression Profile of the $\beta$-catenin Destruction Complex and Histo-pathological Characteristics in Breast Cancer Patients. *, Indicate significance difference between breast cancer patients subgroups, $\mathrm{p}<0.05$ 
( $\mathrm{p}=0.013$ ) gene expression in advance-stage versus earlystage (Figure 2.c and 2.d, respectively). Regarding tumor size, APC gene expression was significant decreased $(\mathrm{p}<0.001)$ in T2 + T3 subgroup (Figure 2.e). Finally, $\beta$-catenin gene expression was significant increased in HER2+ patients $(\mathrm{p}<0.001)$ compared with HER2- patients (Figure 2.f).

The association between rs2240308 and rs151279728 polymorphism and gene expression profile of the $\beta$-catenin destruction complex in breast cancer patients

We determined the AXIN2 variants effect on the gene expression of the $\beta$-catenin destruction complex. We stratified the breast cancer group as patients with ancestral allele and patients with variant allele for rs2240308 and rs 151279728 polymorphisms, separately. We found significant increased expression of PP2A gene associated with variant allele subgroup for $\mathrm{rs} 2040308(\mathrm{p}=0.004)$ and rs151279728 ( $\mathrm{p}=0.013$ ) polymorphisms. Additionally, the breast cancer group was stratified according rs2240308 + rs151279728 polymorphisms combination in wild genotype patients for two polymorphisms and patients with at least a variant allele. Our analyses showed a significant increase of PP2A gene expression level for variant allele subgroup $(\mathrm{p}=0.004)$. No significant association was found between APC, AXIN2, CK1 $\alpha$, $\beta$-catenin and GSK3 $\beta$ gene expression and rs 2240308 and rs151279728 polymorphisms.

\section{Discussion}

The Wnt pathway plays an important role in the carcinogenesis process, as part of proliferation, differentiation and cell morphogenesis processes; alterations of Wnt pathway have been linked to different cancers, including breast cancer (Howe and Brown, 2004; Polakis, 2007; Clevers and Nusse, 2012; Khalil et al., 2012). Genetic alterations of Wnt pathway components were strongly associated with different pathologies and particularly AXIN2 have been associated with dental agenesis (Lammi et al., 2004; Mostowska et al., 2006), orofacial clefts (Letra et al., 2009; Han et al., 2014) and cancer (Lustig et al., 2002; Salahshor and Woodgett, 2005; Gunes et al., 2009; Chapman et al., 2011; Alanazi et al., 2013). AXIN2 mutations are infrequent in human primary breast cancer, AXIN2-null mice model presented a high susceptibility to develop cancer after carcinogenic exposure, furthermore, breast tumor showed high invasive capacity (Yook et al., 2006), demonstrated the important role of AXIN2 in carcinogenesis process. AXIN2 is an important negative regulator of Wnt pathway. In absence of Wnt stimulus, AXIN2 induces $\beta$-catenin degradation by the $\beta$-catenin destruction complex, a large multiprotein complex which includes APC, GSK3 $\beta$, CK $1 \alpha$ and PP2A, additionally, AXIN2 is a transcriptional target of $\beta$-catenin as part of a negative feedback loop (Jho et al., 2002; Lustig et al., 2002; Moon et al., 2004; MacDonald et al., 2009). AXIN2 gene is located on chromosome 17q21-25, a region that presents frequently chromosomal rearrangements in breast cancer (Dong et al., 2001; Salahshor and Woodgett, 2005).
In our study, for the first time it was explored the association between rs2240308 and rs151279728 polymorphisms and breast cancer risk in 102 breast cancer patients and 102 healthy controls from São Paulo state, Brazil. The AXIN2 rs 151279728 polymorphism was found previously in adrenocortical tumors (Chapman et al., 2011), human colorectal cell lines (Suraweera et al., 2006) and melanoma (Castiglia et al., 2008). The rs 151279728 polymorphism is an in-frame $12 \mathrm{bp}$ deletion in exon 8 of AXIN2 gene (c.2013_2024del12), and it was not evaluated in peripheral blood of cancer patients to determine the germline or somatic origin at the time. Castiglia et al. in 2008 (Castiglia et al., 2008) detected the rs 151279728 polymorphism in PBMCs and tumoral cell of a melanoma patient suggesting a germline genetic alteration, but the variant was not found in any of the 93 control individuals. A similar study detected a deletion in familial melanoma patients, interestingly, the variant was found in healthy family members who did not develop melanoma, but was not found in 153 unrelated healthy controls (Pedace et al., 2011). In this study, rs151279728 polymorphism was found in 8 out of 102 breast cancer patients and no one of 102 healthy controls, proving evidence of germline origin, besides the strong association of rs151279728 variant and breast cancer risk. We explored the clinic-pathological variables effect into the rs 151279728 polymorphism distribution. A similar trend was observed, compared with overall results; the polymorphism conferred increased risk in early-stage, tumor larger size, ER+, PR+ and HER2- subgroups. The functional consequence of rs 151279728 polymorphism is unclear, but it is located in a hot spot mutation region of exon 8 (Salahshor and Woodgett, 2005), this region is the hypothetical binding site for PP2A, which acts as a positive regulator of the Wnt pathway by dephosphorylation of AXIN2. The rs151279728 variant leads to loss of two threonine amino acid residues that could be the target of PP2A (Willert et al., 1999; Chapman et al., 2011).

The rs2240308 SNP polymorphism is a $\mathrm{C}>\mathrm{T}$ nucleotide change at position 148 of AXIN2 exon1; this variant generates a replacement of proline to serine at codon 50. The rs 2240308 polymorphism (p.Pro50Ser) is located near RGS domain (amino acids 81 to 200), this domain involves the APC-binding site and participates in tumor suppressor function of AXIN2 protein, through the $\beta$-catenin destruction complex assembly and Wnt pathway regulation (Rubinfeld et al., 1997; Kanzaki et al., 2006). Prior studies evaluated the association between rs2240308 polymorphism and cancer, tooth agenesis and orofacial clefts risk. Some reports did not find association between this polymorphism and orofacial clefts, prostate, ovarian, head and neck cancer (Kanzaki et al., 2006; Letra et al., 2009; Pinarbasi et al., 2011; Mostowska et al., 2014). However, Gunnes et al. and Kanzaki et al. evaluated the rs2240308 SNP in Turkish and Japanese populations, respectively, and its association with lung cancer (Kanzaki et al., 2006; Gunes et al., 2009). Both studies linked the rs2240308 variant and lung cancer. The results concluded that the SNP confers protection to lung cancer; TT genotype showed a decreased lung cancer risk. Wang et al. detected the rs 2240308 SNP in 
PBMCs of breast cancer patients from North-America (Wang et al.,2008), but the authors did not find association between rs 2240308 polymorphism and breast cancer risk. Unlike, we found significant difference between breast cancer patients and control group, in respect to genotype distribution, and our results suggest a strong association between $\mathrm{T}$ allele presence (CT or TT genotype) and increased breast cancer risk. We evaluated the clinicpathological variables effect into the rs2240308 SNP distribution. A similar trend was observed, compared with overall results; the polymorphism conferred increased risk even after stratification based on histological type, hormone receptor status, lymph node status and age of on-set. Wang et al., examined others AXIN2 SNPs and reported common results a significantly elevated risk with younger breast cancers patients (Wang et al., 2008), while Alanazi et al. found a significant protective association with younger breast cancers patients (Alanazi et al., 2013). The discrepancy could be due to different study population (sample size, ethnical diversity), suggesting that the association of AXIN2 variants with breast cancer risk could be different between the populations. To our knowledge, this is the first study to report the rs 2240308 and rs151279728 polymorphisms associated to breast cancer risk in Brazilian population.

Expression profile of genes implicated in the $\beta$-catenin destruction complex has not been widely studied in breast cancer patients. Many studies have identified gene expression profile of Wnt pathway components in breast tumors samples (Lehmann et al., 2011; Gabrovska et al., 2012), but not in peripheral blood of breast cancer patients. The destruction complex is a multiprotein assembly; its core components include to itself $\beta$-catenin, APC, AXIN2, CK $1 \alpha$, GSK $3 \beta$ and PP2A. Mutations and expression changes in destruction complex components result in inappropriate stabilization of $\beta$-catenin and Wnt target gene expression in the absence of a Wnt stimulus (Stamos and Weis, 2013). Herein, we explore the expression profile of APC, AXIN2, CK1 $\alpha, \beta$-catenin, GSK3 $\beta$ and PP2A genes in peripheral blood of breast cancer patients. Our analyses revealed that only AXIN2 was significantly up-regulated in breast cancer patients. Prior studies evaluated AXIN2 expression profile in breast cancer tumors. Consistent with our results, AXIN2 expression was identified to be up-regulated in breast cancer tumors (Gabrovska et al., 2012; Lamb et al., 2013) to be expected of a negative regulator of Wnt signaling in breast cancer, according to suggested activation of WNT signaling in breast cancer through the increased expression of key downstream targets such as AXIN2 (Lustig et al., 2002; Howe and Brown, 2004). Gabrovska et al. proposed the possibility that an up-regulated AXIN2 may still exert a level of growth regulation in cancers, but perhaps not to a level sufficient to completely arrest growth (Gabrovska et al., 2012).

The $\beta$-catenin gene was up-regulated in HER $2+$ patients $(p<0.001)$. This agrees with the observed by Tudoran et al. that found up-regulated $\beta$-catenin in mammary tumors HER2+ when compared to HER2- (Tudoran et al., 2014). In contrast, previous studies reported down-regulated $\beta$-catenin associated to HER2- tumors, suggesting that
HER 2 expression on primary tumors induces differential expression pattern in the peripheral blood of breast cancer patients (Jonsson et al., 2000). The APC is another core component of destruction complex. We found downregulated APC expression in breast cancer patients with advance-stage and larger size tumors. A plausible explication could be the tumor suppressor role of APC, which acts as a negative regulator of the Wnt pathway and other non-Wnt related functions (Nelson and Nathke, 2013). The GSK3 $\beta$ was down-regulated in larger size tumors subgroup. GSK3 $\beta$-mediated phosphorylation of $\beta$-catenin affects its protein concentration, localization and function. GSK3 $\beta$ may be often inactivated in most cancers and deemed a tumor suppressor (Kao et al., 2014). Opposed to our results, Lamb et al. evaluated the GSK3 $\beta$ expression profile in breast cancer tumors found no difference in gene expression, neither association with receptor estrogen status or histological type (Lamb et al., 2013). To our knowledge, for the first time it is reported $\mathrm{CK} 1 \alpha$ and PP2A expression in breast cancer samples. We observe down-regulation of $\mathrm{CK} 1 \alpha$ and PP2A in no-ductal carcinoma patients, suggesting a different molecular signature in blood for each breast cancer histological type. Consistent with priors studies, CK1 $\alpha$ showed decrease expression in lung carcinoma cells, indicating that it may be involved in carcinogenesis process (Xie et al., 2003; Srivastava et al., 2012) and PP2A was down-regulated in clear cell renal cell carcinoma and prostatic cancer. The authors found PP2A decreased expression associated with cancer risk (Westermarck and Hahn, 2008; Li et al., 2014). Sablina et al. demonstrated that decreased activity of any PP2A subunits might contribute to the proliferation of cancer cells, acting as a tumor suppressor gene (Sablina et al., 2007).

In conclusion, this is the first study to associate rs 2240308 (c.148C > T) and rs151279728 (c.2013_2024del12) polymorphisms with breast cancer risk, moreover, we reported for first time $\beta$-catenin destruction complex expression profile in peripheral blood from breast cancer patients. We found AXIN2 genetic alterations associated with higher risk of developing breast cancer and disturbance in $\beta$-catenin destruction complex expression profile associated to breast cancer. The results provide additional support to the role of $\mathrm{Wnt} / \beta$-catenin pathway dysfunction in breast cancer tumorigenesis. Nevertheless, studies to investigate functional consequence of AXIN2 variants are still required. Further investigations into the exact role of AXIN2 in breast cancer development are needed in all breast cancers types and stages.

\section{References}

Alanazi MS, Parine NR, Shaik JP, et al (2013). Association of single nucleotide polymorphisms in Wnt signaling pathway genes with breast cancer in Saudi patients. PLoS One, $\mathbf{8}$, 59555.

Brito C, Portela MC, Vasconcellos MT (2009). Survival of breast cancer women in the state of Rio de Janeiro, Southeastern Brazil. Rev Saude Publica, 43, 481-9.

Castiglia D, Bernardini S, Alvino E, et al (2008). Concomitant activation of Wnt pathway and loss of mismatch repair function in human melanoma. Genes Chromosomes Cancer, 


$$
\text { 47, 614-24. }
$$

Clevers H, Nusse R (2012). Wnt/beta-catenin signaling and disease. Cell, 149, 1192-205.

Chapman A, Durand J, Ouadi L, et al (2011). Identification of genetic alterations of AXIN2 gene in adrenocortical tumors. $J$ Clin Endocrinol Metab, 96, 1477-81.

Dong X, Seelan RS, Qian C, et al (2001). Genomic structure, chromosome mapping and expression analysis of the human AXIN2 gene. Cytogenet Cell Genet, 93, 26-8.

Gabrovska PN, Smith RA, Tiang T, et al (2012). Development of an eight gene expression profile implicating human breast tumours of all grade. Mol Biol Rep, 39, 3879-92.

Gunes EG, Pinarbasi E, Pinarbasi H, et al (2009). Strong association between lung cancer and the AXIN2 polymorphism. Mol Med Report, 2, 1029-35.

Han Y, Zhou L, Ma L, et al (2014). The axis inhibition protein 2 polymorphisms and non-syndromic orofacial clefts susceptibility in a Chinese Han population. J Oral Pathol Med, 43, 554-60.

Herbst A, Jurinovic V, Krebs S, et al (2014). Comprehensive analysis of beta-catenin target genes in colorectal carcinoma cell lines with deregulated Wnt/beta-catenin signaling. BMC Genomics, 15, 74.

Howe LR, Brown AM (2004). Wnt signaling and breast cancer. Cancer Biol Ther, 3, 36-41.

Jho EH, Zhang T, Domon C, et al (2002). Wnt/beta-catenin/ Tcf signaling induces the transcription of Axin2, a negative regulator of the signaling pathway. Mol Cell Biol, 22, 1172-83.

Jonsson M, Borg A, Nilbert M, et al (2000). Involvement of adenomatous polyposis coli (APC)/beta-catenin signalling in human breast cancer. Eur J Cancer, 36, 242-8.

Kanzaki H, Ouchida M, Hanafusa H, et al (2006). Single nucleotide polymorphism of the AXIN2 gene is preferentially associated with human lung cancer risk in a Japanese population. Int J Mol Med, 18, 279-84.

Kao SH, Wang WL, Chen CY, et al (2014). GSK3beta controls epithelial-mesenchymal transition and tumor metastasis by CHIP-mediated degradation of Slug. Oncogene, 33, 3172-82.

Khalil S, Tan GA, Giri DD, et al (2012). Activation status of Wnt/ ss-catenin signaling in normal and neoplastic breast tissues: relationship to HER2/neu expression in human and mouse. PLoS One, 7, 33421.

Klarmann GJ, Decker A, Farrar WL (2008). Epigenetic gene silencing in the Wnt pathway in breast cancer. Epigenetics, 3, 59-63.

Lamb R, Ablett MP, Spence K, et al (2013). Wnt pathway activity in breast cancer sub-types and stem-like cells. PLoS One, $\mathbf{8}$, 67811.

Lammi L, Arte S, Somer M, et al (2004). Mutations in AXIN2 cause familial tooth agenesis and predispose to colorectal cancer. Am J Hum Genet, 74, 1043-50.

Lehmann BD, Bauer JA, Chen X, et al (2011). Identification of human triple-negative breast cancer subtypes and preclinical models for selection of targeted therapies. J Clin Invest, 121, 2750-67.

Letra A, Menezes R, Granjeiro JM, et al (2009). AXIN2 and CDH1 polymorphisms, tooth agenesis, and oral clefts. Birth Defects Res A Clin Mol Teratol, 85, 169-73.

Li J, Sheng C, Li W, et al (2014). Protein phosphatase-2A is downregulated in patients within clear cell renal cell carcinoma. Int J Clin Exp Pathol, 7, 1147-53.

Lustig B, Jerchow B, Sachs M, et al (2002). Negative feedback loop of Wnt signaling through upregulation of conductin/axin2 in colorectal and liver tumors. Mol Cell Biol, 22, 1184-93.

MacDonald BT, Tamai K, He X (2009). Wnt/beta-catenin signaling: components, mechanisms, and diseases. Dev Cell, 17, 9-26.

Moon RT, Kohn AD, De Ferrari GV, et al (2004). WNT and betacatenin signalling: diseases and therapies. Nat Rev Genet, 5, 691-701.
Mosimann C, Hausmann G, Basler K (2009). Beta-catenin hits chromatin: regulation of Wnt target gene activation. Nat Rev Mol Cell Biol, 10, 276-86.

Mostowska A, Biedziak B, Jagodzinski PP(2006). Axis inhibition protein 2 (AXIN2) polymorphisms may be a risk factor for selective tooth agenesis. J Hum Genet, 51, 262-6.

Mostowska A, Pawlik P, Sajdak S, et al (2014). An analysis of polymorphisms within the Wnt signaling pathway in relation to ovarian cancer risk in a Polish population. Mol Diagn Ther, 18, 85-91.

Nelson S, Nathke IS (2013). Interactions and functions of the adenomatous polyposis coli (APC) protein at a glance. $J$ Cell Sci, 126, 873-7.

Pedace L, Castiglia D, De Simone P, et al (2011). AXIN2 germline mutations are rare in familial melanoma. Genes Chromosomes Cancer, 50, 370-3.

Pinarbasi E, Gunes EG, Pinarbasi H, et al (2011). AXIN2 polymorphism and its association with prostate cancer in a Turkish population. Med Oncol, 28, 1373-8.

Polakis P (2007). The many ways of Wnt in cancer. Curr Opin Genet Dev, 17, 45-51.

Rennoll SA, Konsavage WM, Jr., Yochum GS (2014). Nuclear AXIN2 represses MYC gene expression. Biochem Biophys Res Commun, 443, 217-22.

Rubinfeld B, Robbins P, El-Gamil M, et al (1997). Stabilization of beta-catenin by genetic defects in melanoma cell lines. Science, 275, 1790-2.

Sablina AA, Chen W,Arroyo JD, et al (2007). The tumor suppressor PP2A Abeta regulates the RalA GTPase. Cell, 129, 969-82.

Salahshor S, Woodgett JR (2005). The links between axin and carcinogenesis. J Clin Pathol, 58, 225-36.

Santos RA, Teixeira AC, Mayorano MB, et al (2010). Basal levels of DNA damage detected by micronuclei and comet assays in untreated breast cancer patients and healthy women. Clin Exp Med, 10, 87-92.

Schmittgen TD, Livak KJ (2008). Analyzing real-time PCR data by the comparative C(T) method. Nat Protoc, 3, 1101-8.

Srivastava M, Khurana P, Sugadev R (2012). Lung cancer signature biomarkers: tissue specific semantic similarity based clustering of digital differential display (DDD) data. BMC Res Notes, 5,617 .

Stamos JL, Weis WI (2013). The beta-catenin destruction complex. Cold Spring Harb Perspect Biol, 5, 7898.

Suraweera N, Robinson J, Volikos E, et al (2006). Mutations within Wnt pathway genes in sporadic colorectal cancers and cell lines. Int J Cancer, 119, 1837-42.

Teixeira AC, Dos Santos RA, Poersch A, et al (2009). DNA repair in Etoposide-induced DNA damage in lymphocytes of breast cancer patients and healthy women. Int J Clin Exp Med, 2, 280-8.

Tudoran O, Virtic O, Balacescu L, et al (2014). Differential peripheral blood gene expression profile based on her2 expression on primary tumors of breast cancer patients. PLoS One, 9, 102764.

Wang X, Goode EL, Fredericksen ZS, et al (2008). Association of genetic variation in genes implicated in the beta-catenin destruction complex with risk of breast cancer. Cancer Epidemiol Biomarkers Prev, 17, 2101-8.

Westermarck J, Hahn WC (2008). Multiple pathways regulated by the tumor suppressor PP2A in transformation. Trends $\mathrm{Mol}$ Med, 14, 152-60.

Willert K, Shibamoto S, Nusse R (1999). Wnt-induced dephosphorylation of axin releases beta-catenin from the axin complex. Genes Dev, 13, 1768-73.

Xie HL, Chen ZC, He CM, et al (2003). [Cloning and expression analysis of lung carcinoma related gene HLCDG1]. Ai Zheng, 22, 1014-7.

Yook JI, Li XY, Ota I, et al (2006). A Wnt-Axin2-GSK3beta cascade regulates Snaill activity in breast cancer cells. Nat Cell Biol, 8, 1398-406. 